\title{
Unit Commitment and Generation Dispatch of a Hydropower Plant in a Competitive Electricity Market
}

\author{
Juan I. Pérez, José R. Wilhelmi and Luis A. Arévalo \\ Departamento de Ingeniería Civil: Hidráulica y Energética. \\ ETSI de Caminos, Canales y Puertos. Technical University of Madrid \\ c/ Profesor Aranguren s/n, 28040 Madrid (Spain) \\ Phone number: +0034 91 3366705, e-mail address: jiperez@caminos.upm.es
}

\begin{abstract}
This paper presents a model to solve the short-term scheduling problem of a hydropower plant in a deregulated system, based on dynamic programming techniques. The objective of this model is to maximize the revenue obtained by selling energy in a competitive electricity market. The time horizon of the model is divided into hourly periods and ranges from one day to one week. The proposed model determines both the unit commitment and the power to be generated in each hour of the time horizon. The power is considered as a nonlinear function of the water discharge and the reservoir volume; the dependence of the units and plant operating limits on the available gross head has been taken into account; and the water discharged through the spillway has been also allowed for in the model. This approach has been applied to a practical case study, the results of which are analyzed in detail.
\end{abstract}

Key words. Hydropower plant, competitive electricity market, short-term scheduling, head dependence, dynamic programming.

\section{Introduction}

$\mathrm{T}$ his paper considers a price-taker hydropower plant that sells its energy in the day-ahead electricity market, i.e., it does not have influence on the hourly market clearing prices. Bidding strategies considering the effect of the plant production on the market clearing procedure are therefore outside the scope of this paper.

One of the main difficulties faced by most hydro scheduling models lies in the nonlinear relationship existing among the power generated, the water discharge and the net head. For short-term studies the head dependence is usually neglected, especially in the case of large reservoirs, as their variation throughout the planning time horizon is barely significant. Nevertheless, in Spain, and in many other countries all over the world, there are many hydropower plants associated to a reservoir the regulating capacity of which is weekly, daily or even smaller, as it is the more and more frequent case of small to medium head hydropower plants with a limited and environmentally respectful flooded area. In these cases, the head dependence should be considered in order to obtain realistic results.

Several methods have been used to solve the short-term scheduling of a hydro plant: linear programming [1], mixed integer linear programming [2], nonlinear programming [3] and dynamic programming [4], among others. It should be noted that, depending on the market environment, the plant regulating capacity and whether the power generation system is hydro- or thermal-based, the focus of the models may vary slightly but essentially, most approaches proposed in the literature are applicable in a broad range of cases.

In linear programming based models, the hydro generation characteristic is usually reduced to a single unit performance curve approximated either through a piecewise linear curve [5], or by modeling the so-called local best efficiency points [6], the head dependence being therefore neglected. This dependence has been partially considered in only few mixed integer linear programming based models: in [7] the hydro plant generation characteristic is represented by three unit performance curves, each of which is approximated by a nonconcave piecewise linearization; in $[8,9]$, an iterative procedure, formally known as successive mixed integer linear programming, is presented. The problem in this case is that there is no guarantee that the algorithm converges to an optimal solution.

In nonlinear programming based models it is important to avoid the nonconcavity, or nonconvexity (depending on the considered approach), of the objective function since it may result in multiple local optima. In addition, the management of the unit start-ups and shut-downs by means of binary variables leads in these cases to nonlinear mixed integer programming problems [10], the solution of which has not yet reached the maturity of linear or nonlinear programming methods [11].

Due to the inherent complexity of the problem, it is usual to find in the literature approaches that decompose it into a unit commitment subproblem (UCS) to determine the unit status (on/off), and a generation dispatch subproblem (GDS) to determine the optimal power generation. In [12], the UCS is solved by means of a heuristic approach and Lagrangian relaxation is used to solve the GDS, whereas in [13], a genetic algorithm solves the UCS and Lagrangian relaxation is again used to solve the GDS.

Dynamic programming is probably one of the first methods used to solve hydro scheduling problems, whatever the time horizon or the predominant source of energy in the system. 
Recent studies $[14,15]$ have shown that interest on this technique is still relevant. Although it can easily overcome the nonlinear and nonconcave character of the hydro scheduling problem, it poses difficulties to manage the unit start-ups and shut-downs.

This paper presents a dynamic programming model to solve the short-term scheduling problem of a hydropower plant associated to a reservoir the regulating capacity of which is weekly, daily or even smaller. The model provides both the unit commitment and the generation dispatch by means of a suitable discretization of the feasible region. Due to the short-term planning period, the model has been developed in a deterministic framework in the sense that certain variables, such as the water inflow to the reservoir and the hourly market clearing prices, are represented by their forecasted average values. Also, the reservoir volumes at the end and at the beginning of the planning period are assumed to be determined by a longer term planning procedure. The model has been applied to a practical case study, the results of which will be reported and discussed in detail.

This paper is organized as follows: in section 2, the method used to obtain the plant generation characteristic is briefly described; next, in section 3 the approach proposed to solve the short-term scheduling problem is thoroughly described; in section 4, results from a practical case study are reported and discussed; and finally, main conclusions obtained in this study are presented in section 5 .

\section{Plant generation characteristic}

The plant generation characteristic describes the nonlinear and in general nonconcave relationship existing among the power generated, the water discharge and the net head, and is usually represented by a series of power-discharge curves that correspond to different gross head or reservoir volume values. The procedure used in this paper to obtain the plant generation characteristic is based on the method proposed in [4] to solve the instantaneous load dispatch of a hydro plant. Some aspects of said method have been modified in order to consider the variation of the units operating limits with the gross head and to take into account the possibility that the units might have different permissible ranges of net heads. It should be noted that although there exist several heuristic approaches to describe the power production phenomenon in a hydropower plant [16], so far, no analytical expressions have been found in the technical literature.

The procedure proposed in this paper to obtain points of the plant generation characteristic assumes that the following data are known in advance:

a. The curve that relates the gross head (m) to the reservoir volume $\left(\mathrm{hm}^{3}\right)$. b. The variation of the tailrace elevation that depends on the water discharged, and that is generally considered as a polynomial function of different degrees [12, 17].

c. The coefficient that relates the friction head losses in the penstock to the square of the water discharge, usually given in $\mathrm{s}^{2} / \mathrm{m}^{5}$. It should be noted that throughout this paper it is considered that water is conveyed to the units through a single penstock.

d. The turbine efficiency hill curves of all units of the plant, expressed in terms of unit speed and unit discharge, as well as their runner diameters and synchronous speeds.

The proposed procedure consists of repeating the following steps for as many operating points as possible, these being given by the water discharge and the reservoir volume:

Step 1 . The net water head is calculated by subtracting to the gross head the head losses in the penstock and those due to the variation of the tailrace level. If that value is out of the allowable range of net heads of some unit, this unit will be discarded for the following steps.

$$
\begin{gathered}
h=h_{g}-\Delta h_{p}-\Delta h_{t} \\
\Delta h_{p}=\beta q^{2} \\
\Delta h_{t}=f(q)
\end{gathered}
$$

Step 2. Unit speeds of all available units are calculated by means of the following equation.

$$
n_{I}^{j}=\frac{n_{j} D_{j}}{\sqrt{h}}
$$

Step 3. From the turbine efficiency hill curves and equations (5)-(8), maximum and minimum flow limits of all available units must be calculated. Maximum power output depends on the generator characteristics whereas turbine efficiency is generally forced to be higher than a certain value in order to avoid operational problems such as cavitation or draft tube pressure oscillations.

$$
\begin{gathered}
q_{I}^{j}=\frac{q_{j}}{D_{j}^{2} \sqrt{h}} \\
p_{j}=\gamma \eta_{j} q_{j} h \\
p_{j} \leq p_{j}^{\max } \\
\eta_{j} \geq \eta_{j}^{\min }
\end{gathered}
$$

Step 4. The dynamic programming approach presented in [4] to solve the instantaneous load dispatch of a hydro plant is applied to calculate the maximum power generated by all available units: the feasible region of the problem is divided into as many stages as the number of available units; the available flow at the beginning of each stage is taken as the state variable of the problem; and the water flow through each unit is taken as the decision variable of the problem. Then, the dynamic programming recursion equation (9) is 
solved sequentially backwards from the last stage to the first one, subject to constraints (10)-(12).

$$
\begin{gathered}
F\left(x_{j}\right)=\max _{u_{j}}\left\{\gamma \eta\left(u_{j}\right) u_{j} h+F\left(x_{j+1}\right)\right\} \\
x_{j+1}=x_{j}-u_{j}, \quad \forall j=1, \ldots, n u-1 \\
0 \leq u_{j} \leq q_{j}^{\max } \\
\sum_{j=1}^{n n} u_{j}=q
\end{gathered}
$$

Numerical efficiency in equation (9) $\eta$ is estimated by interpolating in the corresponding turbine efficiency hill curves the unit speed and unit flow values, calculated in turn by substituting $u_{j}$ and $h$ in (4) and (5). The generation characteristic of a hydro plant with four generating units is plotted in Fig. 1.

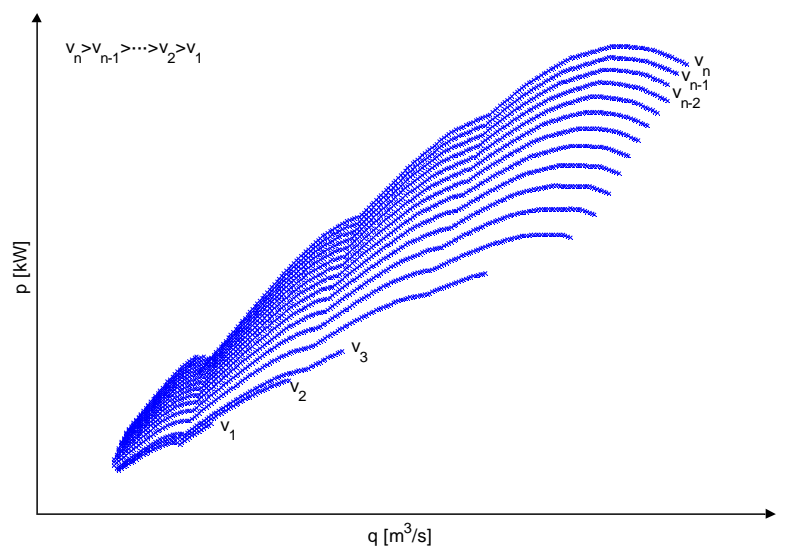

Fig. 1: Generation characteristic of a hydro plant with four generating units.

\section{Short-term scheduling problem}

Once the plant generation characteristic has been obtained, the short-term scheduling problem is solved by means of a novel approach based on dynamic programming. The solution of the problem provides in each stage of the time period considered the optimum values of the following variables: the plant status (on/off); the power output; the reservoir volume; the flow released through the turbines; and the flow spilled through the spillway. It should be noted that the units status (on/off) is implicit in the plant generation characteristic.

\section{A. Discretization of the feasible region}

One of the main contributions of this model lies in the way the feasible region is discretized. This discretization allows to consider the possibility that the plant might be shut-down in any of the stages of the time period considered, as well as that of spilling water over the lip of the spillway. As it was said in the introduction, this approach has been developed in a deterministic framework, i.e., some random variables are represented by their forecasted average values. Such is the case of the water inflow to the reservoir, the forecasted average value of which can vary from one stage to another. The reservoir volumes at the beginning and at the end of the time period considered are in turn assumed to be determined by a longer term planning procedure.

Firstly, the time period is divided into hourly stages. Then, intermediate states of the feasible region are obtained in two different manners, depending on the point, or state, from which the procedure begins.

Forward discretization. Starting from the initial reservoir state, trajectories corresponding both to discharge the absolute maximum flow and to shut-down the plant are plotted sequentially in all stages of the time horizon. At the first stage, the segment defined by the extremes of these trajectories is divided into an integer number of intervals of the same length, $\Delta v$ (see Fig. 2), which define the mesh size of the state diagram. Then, if the reservoir volume surpasses at the end of any stage the maximum volume, this is taken in said stage as the upper limit of the feasible region. On the other hand, when the reservoir volume falls at the end of any stage below the minimum volume, $\Delta v$ is added successively until the reservoir volume is greater or equal than the minimum volume (see Fig. 2). This option allows shutting-down the plant in every stage of the time horizon from any initial state of said stage, whenever feasible, as it can be observed from black trajectories in Fig. 2. If on the contrary, the minimum volume were taken as the lower limit of the feasible region, the resulting mesh (red points in Fig. 2) would not include the states reached when the plant is shut-down, thus eliminating this option.

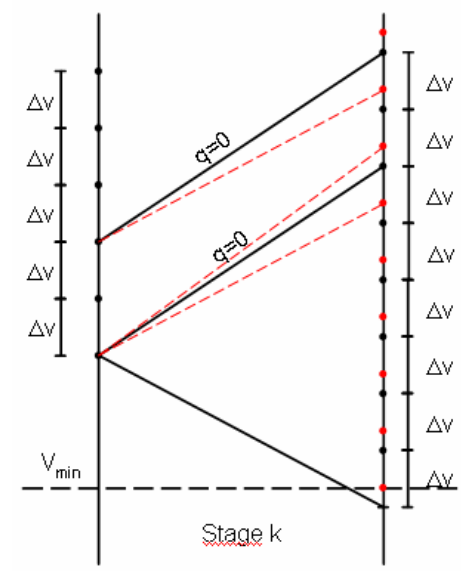

Fig. 2: Obtaining the limits of the feasible region.

Intermediate states at the end of each stage are obtained by adding successively constant volume increments $(\Delta v)$ from the lower limit of the feasible region. The magnitude of this increment depends on the degree of accuracy required. A preliminary, or relaxed, feasible region similar to that shown in Fig. 3 is obtained in this manner. 


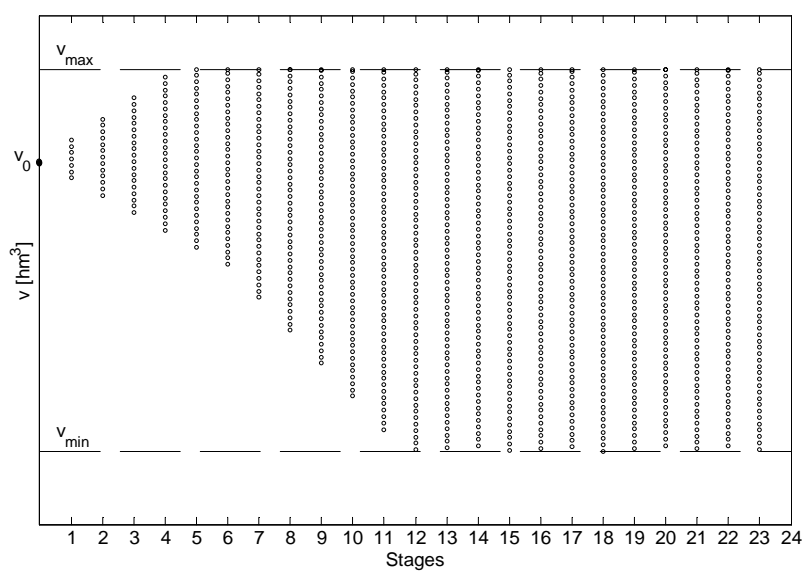

Fig. 3. Preliminary feasible region.

Next, starting from the final reservoir state, trajectories corresponding to discharge the absolute maximum flow and to shut-down the plant are plotted sequentially backwards in all stages of the problem. If the initial state of the trajectory corresponding to shut-down the plant is in any of the stages above the lower limit of the preliminary feasible region, the former is taken as the lower limit of the definitive feasible region. Otherwise, the latter will be taken as the lower limit of the definitive feasible region. All points belonging to the preliminary feasible region that are between the upper and lower limits of the definitive feasible region will also belong to the definitive feasible region. Thus, a definitive feasible region similar to that shown in red in Fig. 4 is obtained.

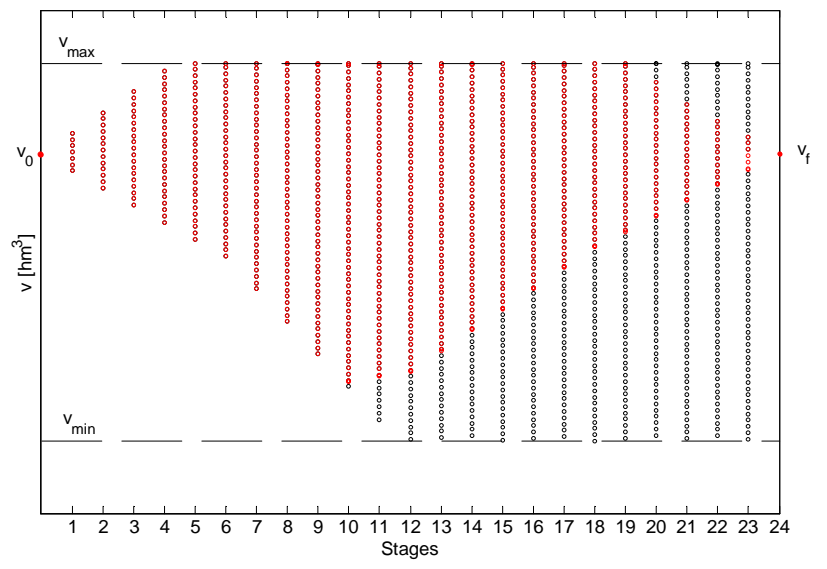

Fig. 4. Definitive feasible region.

Backward discretization. This procedure is identical to the previous one except that it begins from the final reservoir state.

\section{B. Dynamic programming approach}

After obtaining both feasible regions, the following process must be carried out sequentially backwards from the last stage to the first one.
Step 1. Flow and average volume are calculated in each trajectory. It should be noted that the duration of all stages, $\Delta t$, is one hour.

$$
\begin{gathered}
q(i, j, k)=(v(i, k-1)-v(j, k)+c w(k)) / c \\
\bar{v}(i, j, k)=\frac{v(i, k-1)+v(j, k)}{2}
\end{gathered}
$$

Step 2. Maximum and minimum flow limits are obtained in each trajectory by interpolating the average volume in the plant generation characteristic.

Step 3. Power generated is calculated in each trajectory, the following conditions being taken into account:

i. If $q^{\min }(\bar{v}(i, j, k)) \leq q(i, j, k) \leq q^{\max }(\bar{v}(i, j, k)), p(i, j, k)$ is calculated by interpolating in the plant generation characteristic and $s(i, j, k)=0$.

ii. If $q(i, j, k)=0$, the plant is shut-down, so $p(i, j, k)=0$, and $s(i, j, k)=0$.

iii.If $0<q(i, j, k)<q^{\min }(\bar{v}(i, j, k))$ and $v(j, k)=v_{\max }$, a certain amount of water is spilled through the spillway, $s(i, j, k)=q(i, j, k)$.

iv. If $q(i, j, k)>q^{\max }(\bar{v}(i, j, k))$ and $v(j, k)=v_{\max }$, a certain amount of water is discharged through the spillway, $s(i, j, k)=q(i, j, k)-q^{\max }(\bar{v}(i, j, k))$, and $p(i, j, k)$ is calculated by interpolating in the plant generation characteristic.

v. In any other case $p(i, j, k)=-M$ and $s(i, j, k)=0$.

Step 4. Income from selling energy $I(i, j, k)$ are calculated in each trajectory by multiplying the power generated by the energy price $\pi(k)$.

Step 5. The dynamic programming recursion equation (15) is solved to obtain the maximum income accumulated from the initial state $i$ to the final reservoir state $v_{f}$.

$$
\bar{I}(i, k)=\max _{q(i, j, k)}\{I(i, j, k)+\bar{I}(j, k+1)\}
$$

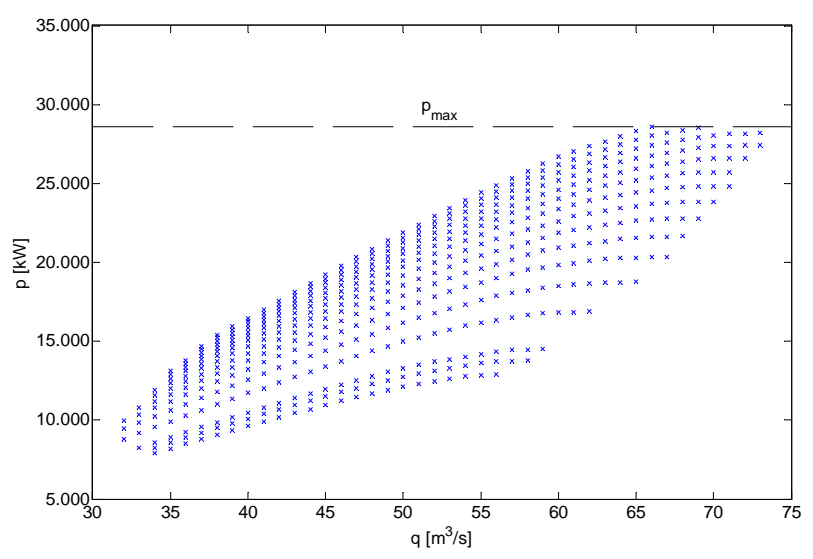

Fig. 5. Case study: Plant generation characteristic. 
The search procedure is to be carried out in both feasible regions: the optimal solution will be the one that provides the maximum income accumulated.

\section{Case study}

The proposed approach has been applied to a realistic case study the results of which are reported and discussed in this section. For the sake of simplicity, the hydro plant analyzed has a single Francis unit the efficiency hill curves of which were taken from [18] (these hill curves have been already used by the authors in [19]). In order to determine the unit operating limits, some criteria presented in [20] were used. The variation of the tailrace elevation is described by a fourth order polynomial function, the coefficients of which have been estimated from those of Jurumirim hydro plant, located in the Paranapanema river, in Brasil (several data from this hydro plant were provided by the company DukeEnergy Brasil). The plant generation characteristic has been obtained following the methodology presented in section 2 and can be seen in Fig. 5 .

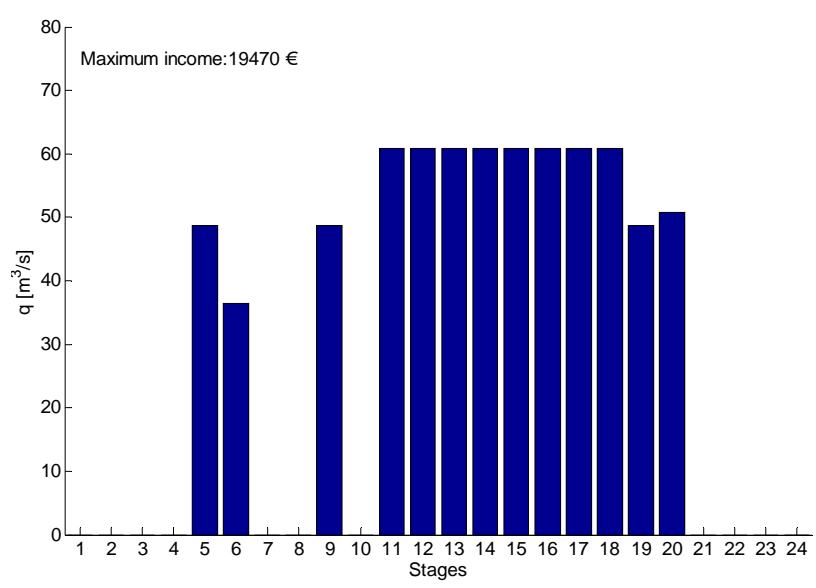

Fig. 6. Results: hourly water flow through the turbine.

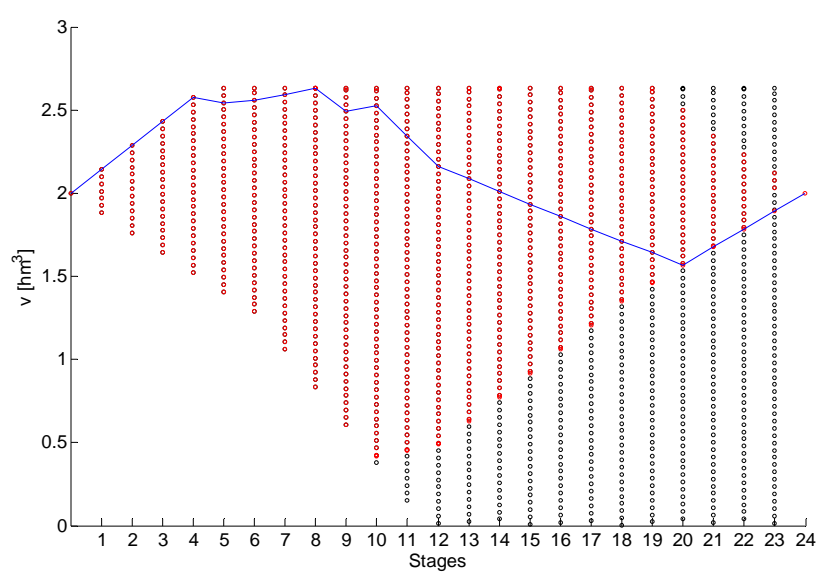

Fig. 7. Results: reservoir trajectory.

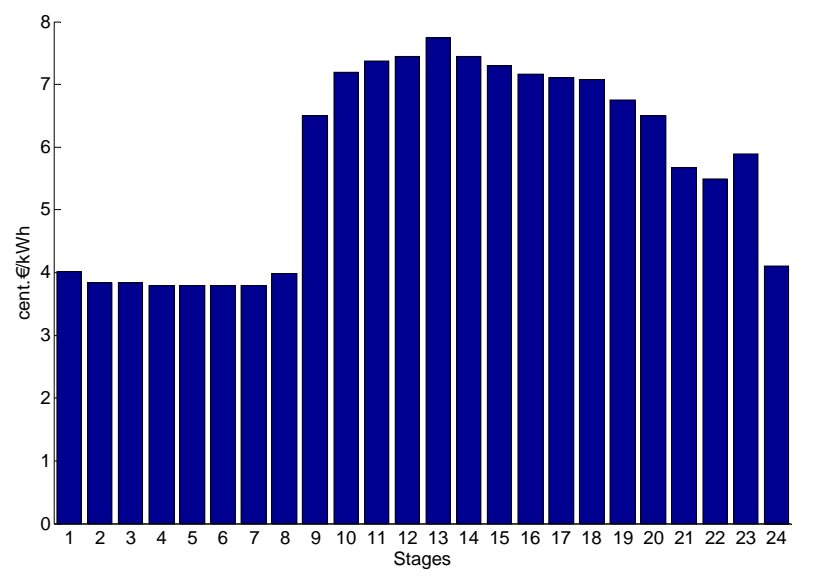

Fig. 8. Hourly energy prices.

The performance of the proposed model has been evaluated under different operating conditions, given by different values of: the forecasted water inflows that vary from one stage to another; and the initial and final reservoir volumes, that were identical in all cases analyzed. The time horizon considered was divided into hourly stages and ranges from one day to one week. The hourly energy prices were taken from the Spanish Market Operator (OMEL) web page [21]. The approach presented in section 3.B has been developed in MATLAB ${ }^{\circledR}$ and has been executed in a Dell PWS 390, Intel dual-core $2.40-\mathrm{GHz}$ processor with $2.00 \mathrm{~GB}$ of RAM. CPU time consumed to reach an optimal solution ranges from few seconds to one minute for daily scheduling and from one to five minutes for weekly scheduling, depending on the number of intermediate states of the feasible region.

Results from one of the cases analyzed can be seen in Figs. 6-8. These figures show respectively: the hourly water flow through the turbine, the reservoir trajectory and the hourly energy prices. As it can be observed in Fig. 7, the reservoir stores water during stages preceding peak hours in order to take advantage of the water head in the latter. During peak hours the reservoir volume decreases rapidly, the maximum flow being released through the turbine, whereas during the last four stages the reservoir stores water again in order to meet the reservoir target level at the end of the day. It is worthwhile to mention that in none of the stages water is spilled through the spillway, thus utilizing all water inflow to produce energy.

\section{Conclusions}

This paper presents a novel approach based on dynamic programming to solve the short-term scheduling of a hydropower plant in a competitive electricity market. This approach provides both the plant status (on/off) and the generation dispatch in each stage of the time horizon considered (from one day to one week). Unit commitment is implicit in the solution of the problem since the plant generation characteristic has been previously obtained by 
solving successively a series of load dispatch problems by dynamic programming.

The generation characteristic considers the power generated as a function of both the water discharge and the reservoir volume as well as the dependence of the units and plant operating limits on the gross head. This is especially important in the case that the regulating capacity of the reservoir associated to the plant is weekly, daily or even smaller. The possibility of spilling water above a certain reservoir volume has been also considered.

Most approaches based on dynamic programming pose difficulties to obtain simultaneously the generation dispatch and the unit commitment. This problem is effectively overcome in this paper by means of a suitable discretization of the feasible region.

\section{Acknowledgement}

Authors want to acknowledge the company Duke-Energy Brasil for providing data from some Brazilian hydro plants.

\section{Appendix-Nomenclature}

The notation used throughout the paper is presented next:

\begin{tabular}{|c|c|}
\hline$h$ & Net water head [m] \\
\hline$h_{g}$ & Gross head [m] \\
\hline$\Delta h_{p}$ & Penstock head losses [m] \\
\hline$\Delta h_{t}$ & Variation of the tailrace level [m] \\
\hline$n_{I}^{j}$ & Unit speed of unit $j[\mathrm{rpm}]$ \\
\hline$n_{j}$ & Synchronous speed of unit $j[\mathrm{rpm}]$ \\
\hline$D_{j}$ & Runner diameter of unit $j[\mathrm{~m}]$ \\
\hline$\beta$ & Friction head losses coefficient $\left[\mathrm{s}^{2} / \mathrm{m}^{5}\right]$ \\
\hline$q$ & Total water discharge $\left[\mathrm{m}^{3} / \mathrm{s}\right]$ \\
\hline$q_{j}$ & Water discharge of unit $j\left[\mathrm{~m}^{3} / \mathrm{s}\right]$ \\
\hline$q_{I}^{j}$ & Unit flow of unit $j\left[\mathrm{~m}^{3} / \mathrm{s}\right]$ \\
\hline$p_{j}$ & Power generated by unit $j[\mathrm{~kW}]$ \\
\hline$\gamma$ & Specific weight of water $\left[9.81 \mathrm{kN} / \mathrm{m}^{3}\right]$ \\
\hline$\eta_{j}$ & Global efficiency of unit [pu] \\
\hline$p_{j}^{\max }$ & Maximum power output of unit $j[\mathrm{~kW}]$ \\
\hline$\eta_{j}^{\min }$ & Minimum global efficiency of unit $j[\mathrm{pu}]$ \\
\hline$F\left(x_{j}\right)$ & Maximum power output from state $x_{j}[\mathrm{~kW}]$ \\
\hline$x_{j}$ & $\begin{array}{l}\text { State variable of the load dispatch problem } \\
{\left[\mathrm{hm}^{3}\right]}\end{array}$ \\
\hline$u_{j}$ & $\begin{array}{l}\text { Decision variable of the load dispatch problem } \\
{\left[\mathrm{m}^{3} / \mathrm{s}\right]}\end{array}$ \\
\hline$q_{j}^{\max }$ & Maximum water discharge of unit $j\left[\mathrm{~m}^{3} / \mathrm{s}\right]$ \\
\hline nu & Number of available units \\
\hline$q(i, j, k)$ & Flow in trajectory $i-j$ during stage $k\left[\mathrm{~m}^{3} / \mathrm{s}\right]$ \\
\hline$v(i, k)$ & $\begin{array}{l}\text { Reservoir volume in state } i \text { at the end of stage } k \\
{\left[\mathrm{hm}^{3}\right]}\end{array}$ \\
\hline$c$ & Conversion factor $\left[0.0036 \mathrm{hm}^{3} / \mathrm{h} / \mathrm{m}^{3} / \mathrm{s}\right]$ \\
\hline$w(k)$ & Water inflow to the reservoir in stage $k\left[\mathrm{~m}^{3} / \mathrm{s}\right]$ \\
\hline $\bar{v}(i, j, k)$ & $\begin{array}{l}\text { Average reservoir volume in trajectory } i-j \\
\text { during stage } k\left[\mathrm{hm}^{3}\right]\end{array}$ \\
\hline
\end{tabular}
$q^{\min }(i, j, k)$ Minimum flow of the plant in trajectory $i-j$ during stage $k\left[\mathrm{~m}^{3} / \mathrm{s}\right]$
$q^{\max }(i, j, k)$ Maximum flow of the plant in trajectory $i-j$ during stage $k\left[\mathrm{~m}^{3} / \mathrm{s}\right]$
$p(i, j, k) \quad$ Plant power output in trajectory $i-j$ during stage $k[\mathrm{~kW}]$
$s(i, j, k) \quad$ Water spilled through the spillway in trajectory $i$-j during stage $k\left[\mathrm{~m}^{3} / \mathrm{s}\right]$
$v_{\max } \quad$ Maximum reservoir volume $\left[\mathrm{hm}^{3}\right]$
$M \quad$ Arbitrary positive real number great enough to discard the corresponding trajectory
$I(i, j, k) \quad$ Income from selling energy in trajectory $i-j$ during stage $k[€]$
$\pi(k) \quad$ Energy price during stage $k[€]$
$\bar{I}(i, k) \quad$ Maximum income accumulated from state $i$ at the beginning of stage $k$ [ $€$.

\section{References}

[1] A.J. Wood and B.F. Wollenberg, Power Generation, Operation and Control, $2^{\text {nd }}$ ed., New York: John Wiley \& Sons, 1996.

[2] G. W. Chang et al., "Experiences with mixed integer linear programming based approaches on short-term hydro scheduling”, IEEE Trans. Power Syst., vol. 16, pp. 743-749, November 2001.

[3] J.P.S. Catalao et al., "Parameterisation effect on the behaviour of a head-dependent hydro chain using a nonlinear model", Electric Power Systems Research, vol. 76, pp. 404-412, 2006.

[4] R. B. Allen and S. G. Bridgeman, "Dynamic programming in hydropower scheduling”, ASCE Journal of Water Resources Planning and Management, vol. 112, pp. 339-353, July 1986.

[5] M. R. Piekutowski, T. Litwinowicz and R. J. Frowd, "Optimal short-term scheduling for a large scale-cascaded hydro system”, IEEE Trans. Power Syst., vol. 9, pp. 805-811, May 1994.

[6] N. Tufegdzic, R.J. Frowd and W.O. Stadlin, “A coordinated approach for real-time short-term hydro scheduling”, IEEE Trans. Power Syst., vol. 11, pp. 1698-1704, November 1996.

[7] A. J. Conejo, J. M. Arroyo, J. Contreras and F. Apolinar Villamor, "Self-scheduling of a hydro producer in a poolbased electricity market”, IEEE Trans. Power Syst., vol. 17, pp. 1265-1272, November 2002.

[8] J. García-González et al. "Under-relaxed iterative procedure for feasible short-term scheduling of a hydro chain", in Proc. of IEEE PowerTech Conference, Bologna, Italy, June 23-26, 2004.

[9] O. B. Fosso and M. M. Belsnes, "Short-term hydro scheduling in a liberalized power system”, in Proc. of IEEE PowerCon, Singapore, November 21-24, 2004.

[10] E. C. Finardi and E.L. da Silva, "Unit commitment of single hydroelectric plant”, Electric Power Systems Research, vol. 75, pp. 116-123, 2005.

[11] R.S.V. Teegavarapu and S.P. Simonovic, "Short-term operation model for coupled hydropower reservoirs", ASCE Journal of Water Resources Planning and Management, vol. 126, no. 2, pp.98-106, March/April 2000.

[12] S. Soares, T. Ohishi, M. Cicogna and A. Arce, "Dynamic dispatch of hydro generating units", in Proc. of IEEE PowerTech Conference, Bologna, Italy, June 23-26, 2004. 
[13] E.F. Santos and T. Ohishi, "A hydro unit commitment model using genetic algorithm”, in Proc. of IEEE Congress on Evolutionary Computation, Portland, Oregon, June 19-23, 2004.

[14] A. Arce, T. Ohishi and S. Soares, "Optimal dispatch of generating units of the Itaipú hydroelectric plant”, IEEE Trans. Power Syst., vol. 17, pp. 154-158, February 2002.

[15] J. Yi, J.W. Labadie and S. Stitt, "Dynamic optimal unit commitment and loading in hydropower systems", ASCE Journal of Water Resources Planning and Management, vol. 129, no.5, September 2003.

[16] M. E. El-Hawari, "Optimal parameter estimation for hydroplant performance models in economic operation studies", IEEE Trans. Power Syst., vol. PWRS-1, pp. 126-131, November 1986.

[17] S. Soares and C.T. Salmazo, "Minimum loss predispatch model for hydroelectric power systems", IEEE Trans. Power Syst., vol. 12, pp. 805-811, August 1997.

[18] C. Mataix, Turbomáquinas hidráulicas, Madrid: ICAI, 1975.

[19] J.I. Pérez and J.R. Wilhelmi, "Nonlinear self-scheduling of a single unit small hydro plant in the day-ahead electricity market”, in Proc. of ICREPQ'07, paper no. 254, Sevilla, Spain, March 2007.

[20] US Department of Interior: Bureau of Reclamation. Selecting hydraulic turbines. A Water Resources Technical Publication, Engineering Monograph no. 20, 1976.

[21] Operador del Mercado Ibérico de Energía - Polo Español, S.A [Online]. www.omel.es 